\title{
Is Quantum Bit Commitment Really Possible?
}

\author{
Hoi-Kwong Lo* and H. F. Chau ${ }^{\dagger}$ \\ School of Natural Sciences, Institute for Advanced Study, Olden Lane, Princeton, New Jersey 08540
}

(Received 8 March 1996)

\begin{abstract}
We show that all proposed quantum bit commitment schemes are insecure because the sender, Alice, can almost always cheat successfully by using an Einstein-Podolsky-Rosen-type of attack and delaying her measurement until she opens her commitment. [S0031-9007(97)02967-0]
\end{abstract}

PACS numbers: $89.70 .+\mathrm{c}, 03.65 . \mathrm{Bz}, 89.80 .+\mathrm{h}$

Work on quantum cryptography was started by Wiesner in a paper written in about 1970, but remained unpublished until 1983 [1]. Recently, there have been lots of renewed activities on the subject. The most well-known application of quantum cryptography is the so-called quantum key distribution $(\mathrm{QKD})$ [2-4], which is useful for making communications between two users totally unintelligible to an eavesdropper. QKD takes advantage of the uncertainty principle of quantum mechanics: Measuring a quantum system in general disturbs it. Therefore, eavesdropping on a quantum communication channel will generally leave unavoidable disturbance in the transmitted signal which can be detected by the legitimate users. Besides QKD, other quantum cryptographic protocols [5] have also been proposed. In particular, it is generally believed [4] that quantum mechanics can protect private information while it is being used for public decision. Suppose Alice has a secret $x$ and Bob a secret $y$. In a "two-party secure computation" (TPSC), Alice and Bob compute a prescribed function $f(x, y)$ in such a way that nothing about each party's input is disclosed to the other, except for what follows logically from one's private input and the function's output. An example of the TPSC is the millionaires' problem: Two persons would like to know who is richer, but neither wishes the other to know the exact amount of money he/ she has.

In classical cryptography, TPSC can be achieved either through trusted intermediaries or by invoking some unproven computational assumptions such as the hardness of factoring large integers. The great expectation is that quantum cryptography can get rid of those requirements and achieve the same goal using the laws of physics alone. At the heart of such optimism has been the widespread belief that unconditionally secure quantum bit commitment (QBC) schemes exist [6]. Here we put such optimism into very serious doubt by showing that all proposed QBC schemes are insecure: A dishonest party can exploit the nonlocal Einstein-Podolsky-Rosen-type correlations [18] in quantum mechanics to cheat successfully. To do so, she generally needs to maintain the coherence of her share of a quantum system by using a quantum computer. We remark that all proposed QBC schemes contain an invalid implicit assumption that some measurements are performed by the two participants. This is why this EPRtype of attack was missed in earlier analysis.
Let us first introduce bit commitment. A bit commitment scheme generally involves two parties, a sender, Alice, and a receiver, Bob. Suppose that Alice has a bit ( $b=0$ or 1$)$ in mind, to which she would like to be committed towards Bob. That is, she wishes to provide Bob with a piece of evidence that she has already chosen the bit and that she cannot change it. Meanwhile, Bob should not be able to tell from that evidence what $b$ is. At a later time, however, it must be possible for Alice to open the commitment. In other words, Alice must be able to show Bob which bit she has committed to and convince him that this is indeed the genuine bit that she had in mind when she committed.

A concrete example of an implementation of bit commitment is for Alice to write down her bit on a piece of paper, which is then put in a locked box and handed over to Bob. While Alice cannot change the value of the bit that she has written down without the key to the box, Bob cannot learn it himself. At a later time, Alice gives the key to Bob, who opens the box and recovers the value of the committed bit. This illustrative example of implementation is, however, is inconvenient and insecure. A locked box may be very heavy and Bob may still try to open it by brute force (e.g., with a hammer).

What do we mean by cheating? As an example, a cheating Alice may choose a particular value of $b$ during the commitment phase and tell Bob another value during the opening phase. A bit commitment scheme is secure against a cheating Alice only if such a fake commitment can be discovered by Bob. For concreteness, it is instructive to consider a simple QBC protocol due to Bennett and Brassard [2]. Its procedure goes as follows: Alice and Bob first agree on a security parameter, a positive integer $s$. The sender, Alice, chooses the value of the committed bit, $b$. If $b=0$, she prepares and sends Bob a sequence of $s$ photons each of which is randomly chosen to be either horizontally or vertically polarized. Of course, the value of $b$ is kept secret during the commitment phase. Moreover, the actual polarization of each photon chosen by Alice is not announced to Bob. Similarly, if $b=1$, she prepares and sends Bob a sequence of $s$ photons each of which is randomly chosen to be either $45^{\circ}$ or $135^{\circ}$ polarized but once again the actual polarization of each photon is kept secret by Alice. Bob chooses randomly between the rectilinear (horizontal and vertical) and diagonal $\left(45^{\circ}\right.$ or $\left.135^{\circ}\right)$ 
bases to measure the polarization of each photon. This completes the commitment phase. A simple calculation shows that the two density matrices describing the $s$ photons corresponding to $b=0$ and $b=1$, respectively, are exactly the same (and are proportional to the identity matrix). Consequently, Bob cannot learn anything about the value of $b$.

At a later time, Alice may open her commitment by announcing the value of $b$ and the actual polarization of each of the $s$ photons. Since Bob has chosen his basis (rectilinear or diagonal) of measurement randomly for each photon in the commitment phase, on average, only half of the $s$ photons have been measured by him in the correct basis. For those photons, Bob can verify that Alice's announced polarizations match his measurement results. Baring EPR attacks, a cheating Alice may, for example, send rectilinear photons in the commitment phase (hence commits to $b=0$ ) but tell Bob that they are diagonal photons in the opening phase (hence announces $b=1$ ). This is cheating. Alice then has to make a random guess for the polarizations of the photons that Bob has measured along the diagonal basis. Since Bob, on average, measures $s / 2$ photons along the diagonal basis, Alice, with such a cheating strategy, has only a probability of $(1 / 2)^{s / 2}$ for success. See [7] for details.

A key weakness of Bennett and Brassard's scheme is that Alice can always cheat successfully by using EPR pairs. Alice can prepare $s$ EPR-pairs of photons and send a member of each pair to Bob during the commitment phase. She skips her measurements and decides on the value of $b$ only at the beginning of the opening phase. If she chooses the value of $b$ to be 0 , she measures the polarization of the photons in her share along the rectilinear basis. It is a standard property (the EPR paradox) of an EPR pair that Alice's measurement result on a photon will always be perpendicular to Bob's result on the other photon of the pair. Alice can, therefore, proudly announce those polarizations. Similarly, for $b=1$, she simply measures along the diagonal basis and proceeds in a similar manner. There is no way for Bob to detect this attack.

Bennett and Brassard noted this weakness in the same paper in which they proposed their scheme [2]. Nonetheless, new QBC schemes have been proposed and it has been generally accepted in the literature $[4,7,8]$ that they defeat an EPR-type of attack. Our goal here is to demonstrate that, contrary to popular belief, precisely the same type of EPR attack defeats all proposed QBC schemes.

All proposed schemes involve only one-way communications from Alice to Bob. On the conceptual level, they all involve Alice sending two quantum systems to Bob, one during the commit phase and the other during the opening phase. (There is no loss of generality in our analysis in considering quantum communications alone since classical communications is just a special case of quantum communications.) More precisely, the general procedure of any proposed QBC scheme can be rephrased in the following manner.

(1) Alice chooses the value of a bit $b$ to which she would like to be committed towards Bob. If $b=0$, she prepares a state

$$
|0\rangle=\sum_{i} \alpha_{i}\left|e_{i}\right\rangle_{A} \otimes\left|\phi_{i}\right\rangle_{B},
$$

where $\left\langle e_{i} \mid e_{j}\right\rangle_{A}=\delta_{i j}$ but the normalized states $\left|\phi_{i}\right\rangle_{B}$ 's are not necessarily orthogonal to each other. Similarly, if $b=1$, she prepares a state

$$
|1\rangle=\sum_{j} \beta_{j}\left|e_{j}^{\prime}\right\rangle_{A} \otimes\left|\phi_{j}^{\prime}\right\rangle_{B},
$$

where $\left\langle e_{i}^{\prime} \mid e_{j}^{\prime}\right\rangle_{A}=\delta_{i j}$ but $\left|\phi_{j}^{\prime}\right\rangle_{B}$ 's are not necessarily orthogonal to each other.

Both Alice and Bob are supposed to know the states $|0\rangle$ and $|1\rangle$. This implies, in particular, that both of them know the states $\left|\phi_{i}\right\rangle_{B}$ and $\left|\phi_{j}^{\prime}\right\rangle_{B}$.

(2) An honest Alice is now supposed to make a measurement on the first register and determine the value of $i$ if $b=0(j$ if $b=1)$.

(3) Alice sends the second register to Bob as a piece of evidence for her commitment.

(4) At a later time, Alice opens the commitment by declaring the value of $b$ and of $i$ or $j$.

(5) Bob performs measurements on the second register to verify that Alice has indeed committed to the genuine bit. More precisely, the data received from Alice (the values of $b$ and also $i$ or $j$ ) should be correlated with Bob's experimental results on the second register. If such expected correlations do appear, Bob accepts that Alice has executed the protocol honestly. Otherwise, Bob suspects that Alice is cheating.

We emphasize that all proposed QBC schemes follow the five-step procedure described above. For instance, Bennett and Brassard's scheme described earlier falls into this class if we give Bob the liberty to store up his photons and measure them only after the opening (step 4) of the commitment by Alice. But, if Alice can cheat against even such a powerful Bob, clearly she can cheat against Bob who has no such storage capability.

Our proof of insecurity of QBC goes as follows: First of all, in order that Bob cannot tell what $b$ is, the second register (the quantum system that Bob receives during the commit phase) must contain very little information about which bit Alice has committed to. As a start, let us consider the ideal case in which the second register contains absolutely no information about the value of $b$. (Bennett and Brassard's scheme [2] and Ardehali's scheme [9] are ideal whereas Brassard and Crépeau's scheme [7] and the most well-known BCJL scheme [8] are nonideal. We sill come to the nonideal case near the end of this Letter.) In the ideal case, to ensure that Bob has no information about the committed bit $b$, the density 
matrices describing the second register associated with the bits 0 and 1 are the same, i.e.,

$$
\operatorname{Tr}_{A}|0\rangle\left\langle 0\left|\equiv \rho_{0}^{B}=\rho_{1}^{B} \equiv \operatorname{Tr}_{A}\right| 1\right\rangle\langle 1| .
$$

It then follows from the Schmidt decomposition [19] that

$$
|0\rangle=\sum_{k} \sqrt{\lambda_{k}}\left|\hat{e}_{k}\right\rangle_{A} \otimes\left|\hat{\phi}_{k}\right\rangle_{B}
$$

and

$$
|1\rangle=\sum_{k} \sqrt{\lambda_{k}}\left|\hat{e}_{k}^{\prime}\right\rangle_{A} \otimes\left|\hat{\phi}_{k}\right\rangle_{B},
$$

where $\left\{\left|\hat{e}_{k}\right\rangle_{A}\right\},\left\{\left|\hat{e}_{k}^{\prime}\right\rangle_{A}\right\}$, and $\left\{\left|\hat{\phi}_{k}\right\rangle_{B}\right\}$ are orthonormal bases of the corresponding Hilbert spaces and $\lambda_{k}$ 's are the eigenvalues of the reduced density operator, $\operatorname{Tr}_{A}|0\rangle\langle 0|=$ $\operatorname{Tr}_{A}|1\rangle\langle 1|$. Notice that the $\lambda_{k}$ 's and $\left|\hat{\phi}_{k}\right\rangle_{B}$ 's are the same for the two states and the only difference lies in Alice's system $\left|\hat{e}_{k}\right\rangle_{A}$ 's vs $\left|\hat{e}_{k}^{\prime}\right\rangle_{A}$ 's. Now consider the unitary transformation $U_{A}$ which maps $\left|\hat{e}_{k}\right\rangle_{A}$ to $\left|\hat{e}_{k}^{\prime}\right\rangle_{A}$. It clearly maps $|0\rangle$ to $|1\rangle$. Note that the transformation $U_{A}$ acts on Alice's system alone and yet rotates $|0\rangle$ to $|1\rangle$. That is, Alice can apply $U_{A}$ without Bob's help. Therefore, Alice can cheat by changing $b=0$ to $b=1$ in the opening phase.

More concretely, consider the following cheating strategy: In the first step, Alice always prepares $|0\rangle$ corresponding to $b=0$. She then skips the second (measurement) step and sends the second register to Bob as prescribed in the third step. She decides on the value of $b$ to announce only in the beginning of the opening phase (step 4). Should she now choose $b$ to be zero, she executes the protocol honestly. On the other hand, if she now chooses $b$ to be one, she applies the unitary transformation $U_{A}$ to rotate $|0\rangle$ to $|1\rangle$ and executes the protocol for $b=1$ instead. Consequently, Alice can always cheat successfully. Notice that Alice is able to cheat primarily because she can delay her measurement until step four. To do so, Alice generally needs a quantum computer. While it is a challenging technological feat to build a quantum computer, it is not forbidden by the laws of quantum physics. The possibility of a dishonest Alice skipping the second step (i.e., delaying her measurements) was not considered in Ref. [8]. This was the chief reason why earlier researchers came to the erroneous conclusion that the BCJL scheme is provably unbreakable.

In the above discussion, we have assumed the ideal situation in which Bob has absolutely no information about the value of $b$ during the commitment phase and hence the density matrices describing the second register for the two cases $b=0$ and $b=1$ are the same. [See Eq. (3)]. However, Brassard and Crépeau's scheme [7] and the BCJL scheme [8] are nonideal in the sense that they violate Eq. (3) slightly and give Bob some probability of distinguishing between $\rho_{0}^{B}$ and $\rho_{1}^{B}$. Intuition seems to indicate that this is not going to change our conclusion: On the one hand, if Bob has a large probability of distinguishing between the two states, the scheme will be unsafe against a cheating Bob. On the other hand, if Bob has only a very small probability of distinguishing between the two states, clearly the two density matrices $\rho_{0}^{B}$ and $\rho_{1}^{B}$ must be close to each other in some sense and essentially the same physics should apply.

Following Mayers [20], we now consider the nonideal case when $\rho_{0}^{B} \neq \rho_{1}^{B}$. The closeness between two states of $B$ specified by the two density matrices $\rho_{0}^{B}$ and $\rho_{1}^{B}$ is commonly described by the concept fidelity [21] which can be defined in terms of purifications. Imagine a system $A$ attached to Bob's system $B$. There are many pure states $\left|\psi_{0}\right\rangle$ and $\left|\psi_{1}\right\rangle$ on the composite system such that

$$
\operatorname{Tr}_{A}\left(\left|\psi_{0}\right\rangle\left\langle\psi_{0}\right|\right)=\rho_{0}^{B} \quad \text { and } \operatorname{Tr}_{A}\left(\left|\psi_{1}\right\rangle\left\langle\psi_{1}\right|\right)=\rho_{1}^{B} .
$$

The pure states $\left|\psi_{0}\right\rangle$ and $\left|\psi_{1}\right\rangle$ are called the purifications of the density matrices $\rho_{0}^{B}$ and $\rho_{1}^{B}$. The fidelity can be defined as

$$
F\left(\rho_{0}^{B}, \rho_{1}^{B}\right)=\max \left|\left\langle\psi_{0} \mid \psi_{0}\right\rangle\right|,
$$

where the maximization is over all possible purifications, $0 \leq F \leq 1 . F=1$ if and only if $\rho_{0}^{B}=\rho_{1}^{B}$. We remark that for any fixed purification of $\rho_{1}^{B}$, e.g., $|1\rangle$ in Eq. (2), there exists a maximally parallel purification of $\rho_{0}^{B}$ which satisfies Eq. (7).

For nonideal QBC schemes, the fact that Bob has a small probability for distinguishing between $\rho_{0}^{B}$ and $\rho_{1}^{B}$ means that [19]

$$
F\left(\rho_{0}^{B}, \rho_{1}^{B}\right)=1-\delta
$$

for some small $\delta>0$. It then follows from Eqs. (7) and (8) that, for the state $|1\rangle$ given in Eq. (2), there exists a purification $\left|\psi_{0}\right\rangle$ of $\rho_{0}^{B}$ such that

$$
\left|\left\langle\psi_{0} \mid 1\right\rangle\right|=F\left(\rho_{0}^{B}, \rho_{1}^{B}\right)=1-\delta .
$$

The strategy of a cheating Alice for a nonideal bit commitment scheme is the same as before. She prepares the state $|0\rangle$ corresponding to $b=0$ in the first step, skips the second (measurement) step, and sends the second register to Bob as prescribed in the third step. She decides on the value of $b$ only in the beginning of the opening phase (step 4). If she now chooses $b=0$, she simply follows the rule. If she chooses $b=1$, she applies a unitary transformation to the quantum system on her share to obtain the state $\left|\psi_{0}\right\rangle$ which satisfies Eq. (9). Such a unitary transformation exists because, as can be seen in the Schmidt decomposition [19], all purifications $|\phi\rangle_{A B}$ of a fixed density matrix $\rho_{B}$ are related to one another by unitary transformations acting on $A$ alone and $A$ is in Alice's hands. Notice that if Alice had been honest, she would have prepared $|1\rangle$ in the first step instead. [See Eq. (2).] Nonetheless, since $\left|\psi_{0}\right\rangle$ and $|1\rangle$ are so similar to each other [see Eq. (9)], Bob clearly has a hard time in detecting the dishonesty of Alice. Therefore, Alice can cheat successfully with a very large probability. 
We thank helpful discussions with M. Ardehali, C. H. Bennett, G. Brassard, C. Crépeau, D. P. DiVincenzo, L. Goldenberg, R. Jozsa, J. Kilian, D. Mayers, J. Preskill, P. Shor, T. Toffoli, and F. Wilczek after the completion of an earlier version of this Letter. This work is supported in part by DOE Grant No. DE-FG02-90ER40542.

Note added. - The insecurity of the BCJL scheme [8] has also been investigated independently by Mayers [20]. More recently, Mayers [22] has generalized the above result to prove that all quantum bit commitment schemes, including ones that involve two-way (quantum) communications between Alice and Bob, are insecure. The same result and the impossibility of ideal quantum coin tossing are discussed in our recent preprint [23]. The impossibility of some other quantum protocols has recently been demonstrated by Lo [24]. These surprising discoveries constitute a major setback to quantum cryptography. The exact boundary to the power of quantum cryptography remains an important subject for future investigations.

*Present address: BRIMS, Hewlett-Packard Labs, Filton Road, Stoke Gifford, Bristol BS12 6QZ, United Kingdom. Electronic address: hkl@hplb.hpl.hp.com

†Present address: Department of Physics, University of Hong Kong, Pokfulam Road, Hong Kong.

Electronic address: hfchau@hkusua.hku.hk

[1] S. Wiesner, SIGACT News 15, 78 (1983); manuscript written around 1970.

[2] C.H. Bennett and G. Brassard, in Proceedings of IEEE International Conference on Computers, Systems, and Signal Processing (IEEE, New York, 1984), p. 175.

[3] A. K. Ekert, Phys. Rev. Lett. 67, 661 (1991).

[4] For reviews on the subject, see, for example, G.P. Collins, Phys. Today 45, No. 11, 23 (1992); C. H. Bennett, G. Brassard, and A. K. Ekert, Sci. Am. 267, 50 (1992).

[5] For a review, see G. Brassard and C. Crépeau, SIGACT News 27, 13 (1996).

[6] Various quantum bit commitment schemes [2,7-9] have been proposed and at least one of them, the so-called Brassard, Crépeau, Jozsa, and Langlois (BCJL) scheme, is even claimed to be provably unbreakable [8]. Quantum bit commitment is an important protocol from which one can construct quantum coin tossing [2,7-9], quantum oblivious mutual identification [10], and quantum oblivious transfer [11-16]. Kilian [17] has shown that, in classical cryptography, oblivious transfer forms the basis of many other protocols including two-party secure computations [17]. This chain of arguments seems to suggest that quantum bit commitment alone is sufficient for implementing two-party secure computations, thus solving a long-standing problem in cryptography.

[7] B. Brassard and C. Crépeau, in Advances in Cryptology: Proceedings of Crypto '90, Lecture Notes in Computer Science Vol. 537 (Springer-Verlag, Berlin, 1991), p. 49.

[8] G. Brassard, C. Crépeau, R. Jozsa, D. Langlois, in Proceedings of the 34th Annual IEEE Symposium on the Foundation of Computer Science, (IEEE Computer Society Press, Los Alamitos, California, 1993), p. 362.

[9] M. Ardehali, Los Alamos Report No. quant-ph/9505019.

[10] C. Crépeau and L. Salvail, in Advances in CryptologyProceedings of Eurocrypt '95 (Springer-Verlag, Berlin, 1995), p. 133.

[11] C.H. Bennett, G. Brassard, C. Crépeau, and M.-H. Skubiszewska, in Advances in Cryptology: Proceedings of Crypto '91, Lecture Notes in Computer Science Vol. 576 (Springer-Verlag, Berlin, 1992), p. 351.

[12] C. Crépeau, J. Mod. Opt. 41, 2445 (1994).

[13] D. Mayers and L. Salvail, in Proceedings of the Workshop on Quantum Computing and Communication, Gaithersburg, Maryland, 1994 (unpublished).

[14] D. Mayers, in Advances in Cryptology: Proceedings of Crypto '95, Lecture Notes in Computer Sciences Vol. 963 (Springer-Verlag, Berlin, 1995), p. 124.

[15] M. Ardehali, Los Alamos Report No. quant-ph/9512026.

[16] A.C.-C. Yao, in Proceedings of the 26th Symposium on the Theory of Computing, 1995 (ACM, New York, 1995), p. 67.

[17] J. Kilian, in Proceedings of 1988 ACM Annual Symposium on Theory of Computing (ACM, Chicago, 1988), p. 20.

[18] A. Einstein, B. Podolsky, and N. Rosen, Phys. Rev. 47, 777 (1935).

[19] See, for example, the appendix of L.P. Hughston, R. Jozsa, and W. K. Wootters, Phys. Lett. A 83, 14 (1993).

[20] D. Mayers, Los Alamos Report No. quant-ph/9603015 (to be published).

[21] R. Jozsa, Mod. Opt. 41, 2343 (1994).

[22] D. Mayers, following Letter, Phys. Rev. Lett. 78, 3414 (1997).

[23] H.-K. Lo and H.F. Chau, in Proceedings of the Fourth Workshop on Physics and Computation, PhysComp' 96, Boston, 1996 (New England Complex Systems Institute, Boston, 1996), p. 76.

[24] H.-K. Lo, Los Alamos Report No. quant-ph/9611031 (to be published). 